\title{
CONTROLLABILITY OF SEMILINEAR STOCHASTIC EVOLUTION EQUATIONS IN HILBERT SPACE
}

\author{
P. BALASUBRAMANIAM ${ }^{1}$ \\ Gandhigram Rural Institute, Deemed University \\ Department of Mathematics \\ Gandhigram 624 302, Tamil Nadu, India \\ J.P. DAUER \\ University of Tennessee at Chattanooga \\ Department of Mathematics \\ 615 McCallie Avenue, Chattanooga, TN 37403-2598 USA
}

(Received October, 1999; Revised June, 2000)

Controllability of semilinear stochastic evolution equations is studied by using stochastic versions of the well-known fixed point theorem and semigroup theory. An application to a stochastic partial differential equation is given.

Key words: Controllability, Stochastic Evolution Equation, Banach Fixed Point Theorem, Wiener Process.

AMS subject classifications: $93 \mathrm{~B} 05$.

\section{Introduction}

Among the methods employed for the controllability of nonlinear systems in finite and infinite dimensional Banach spaces, fixed point techniques are widely used. Anichini [3], Dauer [7] and Dauer, et. al [9] studied the controllability of classical nonlinear systems by means of Schaefer's theorem, Fan's theorem, and Leray-Schauder's theorem, respectively. Several authors have extended the classical finite dimensional controllability results to infinite dimensional controllability results represented by the evolution equations with bounded and unbounded operators in Banach spaces using semigroup theorem (see $[5,8]$ ).

The semigroup theory gives a unified treatment of a wide class of stochastic parabolic, hyperbolic and functional differential equations, and much effort has been devoted to the study of the controllability results of such evolution equations (see $[20])$. Stochastic control theory is a stochastic generalization of classical control

\footnotetext{
${ }^{1}$ Supported by DST grant No. HR $\backslash \mathrm{OY} \backslash \mathrm{M}-02 \backslash 95$ Govt. of India, New Delhi.
} 
theory. Controllability of nonlinear stochastic systems has been one of the wellknown problems discussed in the literature $[4,22,23]$. If the nonlinear terms does not depend on the probability distribution $\mu(t)$ of the process at time $t$, then the process is determined to be a standard Markov process. There are numerous papers in the literature discussing the stability of such stochastic equations in Hilbert spaces (for details see $[1,14,17]$ ). On the other hand, there are situations where the nonlinear term $f$ depends not only on the state of the process at time $t$ but also on the probability distribution. For example, one may think of an interacting particle system (biological, chemical or physical) in which each particle moves in the space $H$ according to the dynamics described by the equation

$$
\begin{gathered}
d x(t)=[A x(t)+f(x(t), \mu(t))] d t+\sqrt{Q} d w(t), t \in J=0[0, T] \\
\mu(t)=\text { probability distribution of } x(t) \\
x(0)=x_{0}
\end{gathered}
$$

with $\mu(t)$ being replaced by the empirical measure

$$
\mu_{N}(t)=\frac{1}{N} \sum_{k=1}^{N} \delta x_{k}(t)
$$

of the $N$ particles $x_{1}(t), x_{2}(t), \ldots, x_{N}(t)$ at time $t$. In other words, we have a system of $N$ coupled semilinear stochastic evolution equations:

$$
\begin{gathered}
d x_{k}(t)=\left[A x_{k}(t)+f\left(x_{k}(t), \mu_{N}(t)\right)\right] d t+\sqrt{Q} d w_{k}(t), t \in J \\
\mu_{N}(t)=\text { empirical measure given by }(1.2) \\
x_{k}(0)=x_{0}, \quad k=1,2, \ldots, N
\end{gathered}
$$

According to the McKean-Vlasov theory (see [2, 18]), under proper conditions, the empirical measure-valued process $\mu_{N}$ converges in probability as $N$ goes to infinity to a deterministic measure-valued function $\mu$ which corresponds to the probability distribution of the process determined by (1.1). The limiting McKean-Vlasov process has many interesting equilibrium and nonequilibrium asymptotic behaviors (at least in the case $H=R^{n}$ ) and therefore has attracted a lot of research attention in recent years (for more information see $[10,11,13,16]$ ). For example, a stochastic model for drug distribution in a closed biological system with a simplified heart, one organ or capillary bed, and recirculation of the blood with a constant rate of flow, where the heart is considered as a mixing chamber of constant volume was described in [21]. Drug concentration in the plasma in given areas of the system is assumed to be a random function of time. Assume that for $t \geq 0, x_{1}(s, t ; \omega)$ is the concentration in moles per unit volume at points in the capillary at time $t$ and $\omega \in \Omega$, the supporting set of a complete probability measure space $(\Omega, \mathcal{A}, P)$ with $\mathcal{A}$ being the $\sigma$-algebra and $P$ is the probability measure. The heart is considered as a mixing chamber of constant volume given by

$$
V=V_{e} /\left(\ln \left(1+V_{e} / V_{r}\right)\right)
$$

where $V_{r}$ is the residual volume of the heart and $V_{e}$ is the injection volume. It is 
assumed that an initial injection is given at the entrance of the heart resulting in a concentration $x(t), 0 \leq t \leq t_{1}$, of the drug in plasma entering the heart, where $t_{1}$ is the duration of injection. Let the time required for the blood to flow from the heart exit to the entrance of the organ be $\tau>0$, and also let $\tau$ be the time required for blood to flow from the exit of the organ to the heart entrance. Drug concentration in the plasma leaving the heart $x(t ; \omega)$ satisfies the integral equation (see [6])

where

$$
x(t ; \omega)=G(t)+\int_{0}^{t} K(s, x(s ; \omega) ; \omega) d s
$$

$$
\begin{gathered}
G(t)=\int_{0}^{T(t)}(C / V) x(s) d s, \quad T(t)=\left[\begin{array}{cc}
t, & 0 \leq t \leq t_{1} \\
t_{1}, & t \geq t_{1}
\end{array}\right. \\
K(s, x(s ; \omega) ; \omega)=(-C / V)\left\{x(s ; \omega)-x_{1}(1, s-\tau ; \omega)\right\}
\end{gathered}
$$

and $x_{1}(1, s ; \omega)=0$ if $s<0$, where $C$ is the constant volume flow rate of plasma in the capillary bed and $x_{1}(1, s ; \omega)$ is the drug concentration in the plasma leaving the organ at time $s$. The mild solutions are in the form of stochastic integral equations. The main objective of this paper is to derive the controllability conditions of semilinear stochastic evolution equations (1.1) in Hilbert space having the probability measure $\mu(t)$. The Banach fixed point theorem is employed to get the suitable controllability conditions. The considered system is an abstract formulation of stochastic partial differential equations (see [12]).

\section{Preliminaries}

Consider the stochastic evolution equation

$$
\begin{gathered}
\frac{d x(t)}{d t}=A x(t)+(B u)(t)+f(x(t), \mu(t))+\sqrt{Q} \frac{d w(t)}{d t}, \quad t \in J=[0, T] \\
x(0)=x_{0},
\end{gathered}
$$

where $A$ is the infinitesimal generator of a strongly continuous semigroup $\{S(t)$, $t \geq 0\}$ of bounded linear operators in a Hilbert space $H$. The state $x(t)$ takes the values in the Hilbert space $H$, the control function $u$ is given in $L^{2}(J ; U)$, and a Hilbert space of admissible control functions with $U$ as a Hilbert space. $B$ is a bounded linear operator from $U$ into $H$. The function $f$ is an appropriate $H$-valued function defined on $H \times M_{\lambda^{2}}(H), M_{\lambda^{2}}(H)$ denotes a proper subset of probability measures on $H ; \mu(t)$ is a probability distribution of $x(t) ; Q$ is a positive, symmetric, bounded operator on $H$ and $w$ is a given $H$-valued cylindrical Wiener process. Let $\left(\Omega, \mathcal{F},\left\{\mathscr{F}_{t}\right\}, \boldsymbol{P}\right)$ be a complete probability space equipped with a family of nondecreasing sub-sigma algebras. $H$ is a real separable Hilbert space with scalar product $\langle\cdot, \cdot\rangle$ and norm $\|\cdot\| \cdot \mathscr{G}(H)$ denotes the Borel sigma algebra of subsets of $H$ and $M(H)$ is the space of probability measures on $\mathfrak{B}(H)$ carrying the usual 
topology of weak convergence Further, let $C(H), C_{b}(H)$ and $C_{b}^{k}(H)$ denote the space of Borel measurable continuous, bounded continuous, and bounded continuous, respectively, up to and including the $k^{t h}$ Frechet derivative functions on $H$. The notation $\langle\mu, \varphi\rangle$ means $\int_{H} \varphi(x) \mu(d x)$ whenever this integral makes sense. Throughout this paper, let $\lambda(x)=1+\|x\|, x \in H$, and define the Banach space

$$
C_{\rho}(H)=\left\{\varphi \in C(H):|\varphi|_{C_{\rho}(H)}=\sup _{x \in H} \frac{\|\varphi(x)\|}{\lambda^{2}(x)}+\sup _{x \neq y} \frac{\|\varphi(x)-\varphi(y)\|}{\|x-y\|}<\infty\right\} .
$$

For $p \geq 1$, let $M_{\lambda}^{s} p(H)$ be the Banach space of signed measures $m$ on $H$ satisfying

$$
|\mu|_{\lambda} p=\int_{H} \lambda^{p}(x)|| m||(d x)<\infty
$$

where $\|m\|=m^{+}+m^{-}$and $m=m^{+}-m^{-}$is the Jordan decomposition of $m$. Let $M_{\lambda^{2}}(H)=M_{\lambda^{2}}^{s}(H) \cap M(H)$ be the set of probability measures on $\mathscr{B}(H)$. We put on $M_{\lambda^{2}}(H)$ a topology induced by the following metric:

$$
\rho\left(\mu_{1}, \mu_{2}\right)=\sup \left\{\left\langle\varphi, \mu_{1}-\mu_{2}\right\rangle:|\varphi|_{\rho}=\sup _{x \in H} \frac{\|\varphi(x)\|}{\lambda^{2}(x)}+\sup _{x \neq y} \frac{\|\varphi(x)-\varphi(y)\|}{\|x-y\|} \leq 1\right\} \text {. }
$$

Then $F=\left(M_{\lambda^{2}}(H), \rho\right)$ forms a complete metric space, and denote $C(J, F)$ the complete metric space of continuous functions from $J$ to $F$ with the metric

$$
D_{T}\left(\mu_{1}, \mu_{2}\right)=\sup _{t \in J} \rho\left(\mu_{1}(t), \mu_{2}(t)\right), \quad \mu_{1}, \mu_{2} \in C(J, F) .
$$

Let $C\left(J, L^{2}(\Omega, \mathcal{F}, \boldsymbol{P} ; H)\right)$ be the Banach space of continuous maps from $J$ into $L^{2}(\Omega, \mathcal{F}, \boldsymbol{P} ; H)$ satisfying the condition $\sup _{t \in J} E\|x(t)\|^{2}<\infty$. Let $K$ be the closed subspace of $C\left(J, L^{2}(\Omega, \mathscr{F}, \boldsymbol{P} ; H)\right)$ consisting of measurable and $\mathscr{F}_{t}$-adapted processes $x=\{x(t): t \in J\}$. Then $K$ is a Banach space with the norm topology given by

$$
\|x\|_{K}=\left(\sup _{t \in J} E\|x(t)\|^{2}\right)^{1 / 2} \text {. }
$$

For the existence of solution of (2.1) assume the following hypotheses:

(H1) (i) $A$ is the infinitesimal generator of a $C_{0}$-semigroup $\{S(t): t \geq 0\}$ of bounded linear operators on $H$ of negative type

$$
\|S(t)\| \leq C_{1} e^{-\omega t}, \quad t \geq 0
$$

for some positive constants $C_{1}>0$ and $\omega>0$;

(ii) $Q$ is a positive, symmetric, bounded operator in $H$ such that the operator $Q_{t}$ defined by

$$
Q_{t}=\int_{0}^{t} S(r) Q S^{*}(r) d r
$$

is nuclear for all $t \geq 0$ and $\sup \operatorname{tr} Q_{t}<\infty$.

$$
t \in J
$$


(iii) $w$ is an $H$-valued Cylindrical Wiener process defined on $(\Omega, \mathcal{F}, \boldsymbol{P})$ with covariance operator $I$.

(H2) $f: H \times F \rightarrow H$ satisfies the conditions

$$
\left\|f\left(x, \mu_{1}\right)-f\left(y, \mu_{2}\right)\right\| \leq C_{2}\left(\|x-y\|+\rho\left(\mu_{1}, \mu_{2}\right)\right)
$$

and

$$
\|f(x, \mu)\| \leq C_{3}\left(1+\|x\|+|\mu|_{\lambda}\right)
$$

where $C_{2}, C_{3}$ are positive constants.

(H3) The linear operator $W$ from $L^{2}(J ; U)$ into $H$ defined by

$$
W u=\int_{0}^{T} S(T-s) B u(s) d s
$$

has an invertible operator $W^{-1}$ defined on $H \backslash \operatorname{Ker} W$ (see [15]) and there exist positive constants $C_{4}$ and $C_{5}$ such that

$$
\|B\|^{2} \leq C_{4} \text { and }\left\|W^{-1}\right\|^{2} \leq C_{5} .
$$

Suppose the hypotheses (H1) and (H2) are satisfied. Then the stochastic process

$$
\begin{aligned}
x(t)=S(t) x_{0}+ & \int_{0}^{t} S(t-s)[(B u)(s)+f(x(s), \mu(s))] d s \\
& +\int_{0}^{t} S(t-s) \sqrt{Q} d w(s)
\end{aligned}
$$

for $t \in J$ defined on the probability space $(\Omega, \mathcal{F}, \boldsymbol{P})$ is said to be a mild solution in $K$ of equation (2.1) for a given initial data $x_{0}$ (see [1]).

Definition 2.1: The stochastic evolution equation (2.1) is said to be controllable on $J$, if, for every $x(0)=x_{0} \in H$, there exists a control $u \in L^{2}(J ; U)$ such that the solution $x(\cdot)$ of $(2.1)$ satisfies $x(T)=x_{1}$ where $x_{1}$ and $T$ are preassigned terminal state and time, respectively. If the system is controllable for all $x_{0}$ at $t=0$ and for all $x_{1}$ at $t=T$, it is called completely controllable on $J$.

\section{Main Result}

Theorem 3.1: Suppose the hypotheses $(H 1)-(H 3)$ are satisfied; then the system $(2.1)$ is completely controllable on $j$.

Proof: Using the hypothesis (H3), define the control

$$
u(t)=-W^{-1}\left[S(T) x_{0}+\int_{0}^{T} S(T-s) f(x(s), \mu(s)) d s+\int_{0}^{T} S(T-s) \sqrt{Q} d w(s)\right](t) .
$$


Now it is shown that, when using this control, the operator defined by

$$
\begin{gathered}
(\Phi x)(t)=S(t) x_{0}-\int_{0}^{t} S(t-\eta) B W^{-1}\left[S(T) x_{0}\right. \\
\left.+\int_{0}^{T} S(T-s) f(x(s), \mu(s)) d s+\int_{0}^{T} S(T-s) \sqrt{Q} d w(s)\right](\eta) d \eta \\
+\int_{0}^{t} S(t-s) f(x(s), u(s)) d s+\int_{0}^{t} S(t-s) \sqrt{Q} d w(s)
\end{gathered}
$$

has a fixed point. This fixed point is a solution of equation $(2.1)$. Clearly $(\Phi x)(0)=$ $x_{0}$, which means that the control $u$ steers the semilinear evolution equation from the initial state $x_{0}$ to $x_{1}$ in time $T$, provided we can obtain a fixed point of the nonlinear operator $\Phi$.

First, we show that $\Phi$ maps $K$ into $K$, for a fixed measure-valued function $\mu \in$ $C(J, F)$. Since $|a+b+c|^{2} \leq 9\left(|a|^{2}+|b|^{2}+|c|^{2}\right)$ for any real numbers $a, b$ and $c$, we have

$$
\begin{gathered}
\|(\Phi x)(t)\|^{2} \leq 9\left\{E\left(\left\|S(t) x_{0}\right\|^{2}\right)+E \| \int_{0}^{T} S(t-\eta) B W^{-1}\left[S(T) x_{0}\right.\right. \\
\left.\left.+\int_{0}^{T} S(T-s) f(x(s), \mu(s)) d s+\int_{0}^{T} S(T-s) \sqrt{Q} d w(s)\right](\eta) d \eta \|^{2}\right\} \\
9\left\|\int_{0}^{t} S(t-s) f(x(s), \mu(s)) d s\right\|^{2}+9\left\|\int_{0}^{t} S(t-s) \sqrt{Q} d w(s)\right\|^{2} \\
\leq 9 C_{1}^{2}\left\{E\left\|x_{0}\right\|^{2}+T C_{4} C_{5}\left[C_{1}^{2} E\left\|x_{0}\right\|^{2}+T C_{1}^{2} E \int_{0}^{t} C_{3}^{2}\left(1+\|x\|^{2}+|\mu(s)|{ }_{\lambda}^{2}\right) d s\right.\right. \\
\left.\left.+\operatorname{tr} Q_{T}\right]+T E \int_{0}^{t} C_{3}^{2}\left(1+\|x\|^{2}+|\mu(s)|_{\lambda}^{2}\right) d s\right\}+9 \operatorname{tr} Q_{t} \\
\leq 9 C_{1}^{2}\left\{E\left\|x_{0}\right\|^{2}+T C_{4} C_{t}\left[C_{1}^{2} E\left\|x_{0}\right\|^{2}+T^{2} C_{1}^{2} C_{3}^{2}\left(1+\|x\|^{2}+|\mu(s)|_{\lambda}^{2}\right)+\operatorname{tr} Q_{T}\right]\right. \\
\left.+T^{2} C_{3}^{2}\left(1+\|x\|^{2}+|\mu(s)|_{\lambda}^{2}\right)\right\}+9 \operatorname{tr} Q_{T} \\
\leq k_{1}+k_{2}\|x\|_{K}^{2}
\end{gathered}
$$


where

$$
\begin{gathered}
k_{1}=9\left(1+T C_{1}^{2} C_{4} C_{5}\right)\left\{C_{1}^{2} E\left\|x_{0}\right\|^{2}+T^{2} C_{1}^{2} C_{3}^{2}\left(1+\sup _{t \in J}|\mu(s)|_{\lambda}^{2}\right)+\operatorname{tr} Q_{T}\right\} \\
k_{2}=9 T^{2} C_{1}^{2} C_{3}^{2}\left(1+T C_{1}^{2} C_{4} C_{5}\right)
\end{gathered}
$$

are two positive constants.

Hence $\|(\Phi x)(t)\|_{K}^{2}<\infty$ for $x \in K$ and it is easy to see that $(\Phi x)(t)$ is $\mathscr{F}_{t}$-measurable whenever $x(t)$ is $\mathscr{F}_{t}$-measurable and so $\Phi$ maps $K$ into $K$.

To complete the proof, it remains to show that $\Phi x \in C(J, F)$. Let $\Phi x=$ $\{(\Phi x)(t): t \in J\}$, then it is enough to show that $t \rightarrow(\Phi x)(t)$ is continuous, since $x \in K$ and $(\Phi x)(t) \in M_{\lambda^{2}}(H)$ for any $t \in J$. Let $z(t)=\int_{0}^{t} S(t-s) \sqrt{Q} d w(s)$ and applying semigroup property of $S(t)$ for $0 \leq s \leq t \leq T$, we have

$$
\begin{aligned}
& E\|(\Phi x)(t)-(\Phi x)(s)\|^{2} \leq 9 E\left(\left\|S(s)[S(t-s)-I] x_{0}\right\|^{2}\right) \\
& +18 E\left\|\int_{s}^{t} S(t-\eta) B W^{-1}\left[S(T) x_{0}+\int_{0}^{T} S(T-s) f(x(s), \mu(s)) d s+z(T)\right](\eta) d \eta\right\|^{2} \\
& +18 E \| \int_{0}^{s}[S(t-s)-I] S(t-\eta) B W^{-1}\left[S(T) x_{0}\right. \\
& \left.+\int_{0}^{T} S(T-s) f(x(s), \mu(s)) d s+z(T)\right](\eta) d \eta \|^{2} \\
& +18 E\left\|\int_{s}^{t} S(t-\tau) f(x(\tau), \mu(\tau)) d \tau\right\|^{2} \\
& +18 E\left\|\int_{0}^{s}[S(t-s)-I] S(t-\tau) f(x(\tau), \mu(\tau)) d \tau\right\|^{2}+9\|z(t)-z(s)\|^{2} \\
& \leq 9 E\left(\left\|S(s)[S(t-s)-I] x_{0}\right\|^{2}\right) \\
& +18 C_{1}^{2} C_{4} C_{5}(t-s)^{2} E\left\|S(T) x_{0}+\int_{0}^{T} S(T-s) f(x(s), \mu(s)) d s+z(T)\right\|^{2} \\
& +18 E \| \int_{0}^{s}[S(t-s)-I] S(t-\eta) B W^{-1}\left[S(T) x_{0} \int_{0}^{T} S(T-s) f(x(s), \mu(s)) d s\right. \\
& +z(T)](\eta) d \eta \|^{2}+18 C_{1}^{2} C_{3}(t-s) E \int_{0}^{t}\left(1+\|x(\tau)\|^{2}+|\mu(\tau)|_{\lambda}^{2}\right) d \tau
\end{aligned}
$$




$$
18 E\left\|\int_{0}^{s}[S(t-s)-I] S(t-\tau) f(x(\tau), \mu(\tau)) d \tau\right\|^{2}+9\|z(t)-z(s)\|^{2}
$$

Since $S(t)$ is strong continuous (see Pazy [19]), $[S(t-s)-I] h$ converges to 0 as $t \rightarrow s$ for any $h \in H$. One can easily derive by Lebesgue's dominated convergence theorem that the first, third and fifth terms on the right-hand side of equation (3.2) tends to 0 as $t \rightarrow s$. Further, since $z(t)$ is a continuous process, $z(t)-z(s)$ converges to 0 as $t \rightarrow s$ with probability 1 . Lebesgue's dominated convergence theorem and the fact $\operatorname{tr} Q_{t}<\infty$ can be used to claim that

$$
\lim _{t \rightarrow s} E\|z(t)-z(s)\|^{2}=0 .
$$

Thus, $E\|(\Phi x)(t)-(\Phi x)(s)\|^{2}$ converges to 0 as $t \rightarrow s$. For any $\varphi \in C_{\lambda^{2}}(H)$, by definition of the metric $\rho$, we have

$$
\begin{aligned}
\|\langle\varphi,((\Phi x)(t)- & (\Phi x)(s))\rangle\|=\| E[(\varphi x)(t)-(\varphi x)(s)] \| \\
& \leq|\varphi|_{\rho} E\|x(t)-X(s)\|
\end{aligned}
$$

and therefore,

$$
\lim _{t \rightarrow s} \rho((\Phi x)(t),(\Phi x)(s))=0
$$

hence $(\Phi x)(t) \in C(J, F)$. Now we prove that $\Phi$ is a contraction map on $C(J, F)$ and therefore has a unique fixed point. Indeed, for $x, y \in K$ satisfying $x(0)=y(0)$ we have

$$
\begin{gathered}
E\|(\Phi x)(t)-(\Phi y)(t)\|^{2} \\
\leq E\left\|\int_{0}^{t} S(t-\eta) B W^{-1}[S(T-s)[f(x(s), \mu(s))-f(y(s), \mu(s))] d s](\eta) d \eta\right\|^{2} \\
+E\left\|\int_{0}^{t} S(t-s)[f(x(s), \mu(s))-f(y(s), \mu(s))] d s\right\|^{2} \\
\leq\left(1+T C_{1}^{2} C_{4} C_{5}\right) T C_{1}^{2} C_{2} \int_{0}^{t} E\|x(s)-y(s)\|^{2} d s \\
=C_{6} \int_{0}^{t} E\|x(s)-y(s)\|^{2} d s
\end{gathered}
$$

where $C_{6}=\left(1+T C_{1}^{2} C_{4} C_{5}\right) T C_{1}^{2} C_{2}$. For any integer $n \geq 1$, by iterations, it follows that

$$
\left\|\left(\Phi^{n} x\right)(t)-\left(\Phi^{n} y\right)(t)\right\|_{K}^{2} \leq \frac{C_{6}^{n} T^{n}}{n !}\|x(s)-y(s)\|^{2}
$$


Since for sufficiently large $n, \frac{C_{6}^{n} T^{n}}{n !}<1, \Phi^{n}$ is a contraction map on $K$ and therefore $\Phi$ itself has a unique fixed point $x$ in $K$. Any fixed point of $\Phi$ is a solution of (2.1) on $J$ satisfying $(\Phi x)(t)=x(t) \in H$ for all $x_{0}$ and $T$. Thus, the system (2.1) is completely controllable on $J$.

\section{Example}

Consider the following nonlinear stochastic partial differential equation of the form

$$
\begin{gathered}
\partial_{t} x(t, \xi)=\frac{\partial^{2}}{\partial \xi^{2}} x(t, \xi) d t+f\left(\xi, \mu(t),\left\langle x(t, \cdot), h_{1}\right\rangle, \ldots,\left\langle x(t, \cdot), h_{n}\right\rangle\right) d t+(B u)(t) d t \\
+\sum_{k=1}^{\infty} \lambda_{k} \sin (k \xi) d \beta_{k}(t), \xi \in(0, \pi), t>0 \\
x(t, 0)=x(t, \pi)=0, t>0 \\
x(0, \cdot)=x_{0}(\cdot) \in X=H=L^{2}(0, \pi)
\end{gathered}
$$

with the following assumptions given by:

(1) Let $\operatorname{dom} A=H^{2}(0, \pi) \cap H_{0}^{1}(0, \pi)$ and $(A \phi) \xi=\frac{\partial^{2}}{\partial \xi^{2}} \phi(\xi), \xi \in(0, \pi), \phi \in \operatorname{dom} A$ and $B$ is a bounded linear operator from the control space $U=L^{2}(0, \pi)$ into $H$.

(2) Define the function $G: H \rightarrow H$ by choosing $h_{1}, h_{2}, \ldots, h_{n} \in H$ and a function $f:[0, \pi] \times F \times R^{n} \rightarrow R,\left(\xi, \mu, y_{1}, y_{2}, \ldots, y_{n}\right) \rightarrow f\left(\xi, \mu, y_{1}, y_{2}, \ldots, y_{n}\right)$ and setting

$$
(G g)(\xi)=f\left(\xi, \mu,\left\langle g, h_{1}\right\rangle,\left\langle g, h_{2}\right\rangle, \ldots,\left\langle g, h_{n}\right\rangle\right), \xi \in[0, \pi], g \in H
$$

$\langle\cdot, \cdot\rangle$ is the usual scalar product in $H$. Also for $y_{1}, y_{2}, y_{n} \in J$ and $i=1,2, \ldots, n$, assume $f, \frac{\partial f}{\partial \xi}, \frac{\partial f}{\partial y_{i}}, \frac{\partial^{2} f}{\partial \xi \partial y_{i}}$ are bounded
and continuous on $[0, \pi] \times F \times R^{n}$, such that

$$
\begin{gathered}
f\left(0, \mu, y_{1}, y_{2}, \ldots, y_{n}\right)=f\left(\pi, \mu, y_{1}, y_{2}, \ldots, y_{n}\right)=0 \\
{\left[\partial f / \partial y_{i}\right]\left(0, \mu, y_{1}, y_{2}, \ldots, y_{n}\right)=\left[\partial f / \partial y_{i}\right]\left(\pi, \mu, y_{1}, y_{2}, \ldots, y_{n}\right)=0 .}
\end{gathered}
$$

(4) The functions $e_{k}(\xi)=\sqrt{2} / \pi \sin k \xi, \xi \in(0, \pi)$, for an orthonormal basis of $H$ consisting of eigenvectors of $A$ corresponding to the eigenvalues $\alpha_{k}=-k^{2}$, $k=1,2, \ldots$ etc., and $\beta_{k}(t)$ are standard, real independent Wiener processes. Take a sequence of numbers $\left\{\lambda_{k}\right\}$ and define the operator $Q$ by setting $Q e_{k}=\lambda_{k} e_{k}, k=1,2, \ldots$. Assume that $\lambda_{k}>0, \sup _{k} \lambda_{k}<\infty$, $\sup _{k} \lambda_{k}-1 / 2 e^{-t k^{2}}<\infty$ for $t>0$.

Then (4.1) has an abstract formulation of the following nonlinear stochastic equation in a Hilbert space with constant, but possibly degenerate diffusion term

$$
\frac{d x(t)}{d t}=A x(t)+f(x(t), \mu(t))+(B u)(t)+Q^{1 / 2} \frac{d w(t)}{d t}, t \in J .
$$




$$
x(0)=x_{0} \in H
$$

where the linear operator $A$ is the infinitesimal generator of a strongly continuous semigroup $e^{A t}, t \geq 0$ in $H, Q$ is a continuous linear, self-adjoint nonnegative operator in $H$, and the operators defined by

$$
Q_{t} x=\int_{0}^{t} e^{s A} Q e^{s A^{*}} x_{0} d s, x_{0} \in H
$$

are trace class. Further $f: H \rightarrow H$ is Lipschitz continuous and $w(t), t \geq 0$ is a cylindrical Wiener process in $H$. Then 94.2 ) has a unique solution as the following (see [12])

$$
x(t)=e^{t A} x_{0}+\int_{0}^{t} e^{(t-s A}[(B u)(s)+f(x(s), \mu(t))] d s+\int_{0}^{t} e^{(t-s A} Q^{1 / 2} d w(s) .
$$

Hence by Theorem 3.1 , for $S(t)=e^{t A}$, the system (4.1) is completely controllable on $J$.

\section{References}

[1] Ahmed, N.U., Stability of limit measures induced by stochastic differential equations on Hilbert space, Comput. Math. Appl. 22 (1991), 41-48.

[2] Ahmed, N.U. and Ding, X., A semilinear McKean-Vlasov stochastic evolution equation in Hilbert space, Stoch. Proc. Appl. 60 (1995), 65-85.

[3] Anichini, G., Controllability of nonlinear control process with prescribed controls, J. Optim. Theory Appl. 32 (1980), 183-199.

[4] Astrom, K.J., Introduction to Stochastic Control Theory, Academic Press, New York 1970.

[5] Balachandran, K., Dauer, J.P. and Balasubramaniam, P., Controllability of nonlinear integrodifferential systems in Banach space, J. Optim. Theory Appl. 84 (1995), 83-91.

[6] Bellman, R., Jacquez, J. and Kalaba, R., Some mathematical aspect of Chemotherapy. I: One-organ models, Bull. Math. Biophys. 22 (1960), 181-198.

[7] Dauer, J.P., Controllability of nonlinear systems with restrained controls, J. Optim. Theory Appl. 14 (1974), 251-262.

[8] Dauer, J.P. and Balasubramaniam, P., Null controllability of semilinear integrodifferential system in Banach space, Appl. Math. Lett. 10 (1997), 117-123.

[9] Dauer, J.P., Balachandran, K. and Balasubramaniam, P., Asymptotic null controllability of nonlinear perturbed systems, J. Optim. Theory Appl. 83 (1994), $167-179$.

[10] Dawson, D.A., Critical dynamics and fluctuations for a mean-field model of cooperative behavior, J. Statist. Phys. 31 (1983), 29-85.

[11] Dawson, D.A. and Gärtner, J., Large deviations from the McKean-Vlasov limit for weakly interacting diffusions, Stochastics 20 (1987), 247-308.

[12] Fuhrman, M., Smoothing properties of nonlinear stochastic equations in Hilbert spaces, Nonl. Diff. Eqns. Appl. 3 (1996), 445-464. 
[13] Funaki, T., A certain class of diffusion processes associated with nonlinear parabolic equations, Probab. Theory Related Fields 67 (1984), 331-348.

[14] Ichickawa, A., Stability of semilinear stochastic evolution equations, J. Math. Anal. Appl. 90 (1982), 12-44.

[15] Jousis, J.C. and Wexler, D., On exact controllability in Hilbert spaces, J. Diff. Eqns. 49 (1983), 258-269.

[16] Leonard, C., Large deviations and law of large numbers for mean field type interacting particle system, Stoch. Process. Appl. 25 (1987), 215-235.

[17] Liu, K., On stability for a class of semilinear stochastic evolution equations, Stoch. Proc. Appl. 70 (1997), 219-241.

[18] McKean, H.P., A class of Markov processes associated with nonlinear parabolic equations, Proc. NAS 56 (1966), 1907-1911.

[19] Pazy, A., Semigroups of Linear Operators and Applications to Partial Differential Equations, Springer Verlag, New York 1983.

[20] Rabah, R. and Karakchou, J., Exact controllability and complete stabilizability for linear systems in Hilbert spaces, Appl. Math. Lett. 10 (1997), 35-40.

[21] Tsokos, C.P. and Padgett, W.J., Random Integral Equations with Application to Life Sciences and Engineering, Academic Press, New York 1974.

[22] Wonham, V.M., Random Differential Equations in Control Theory, Prob. Meth. in Appl. Math. Vol. 2, Academic Press, New York 1970.

[23] Zabczyk, J., Controllability of stochastic linear systems, stochastic differential systems, In: Lecture Notes in Control and Information Sciences, Springer Verlag, New York 43 (1982), 144-154. 


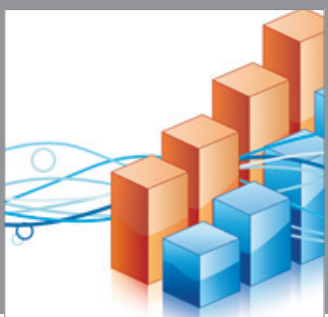

Advances in

Operations Research

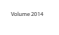

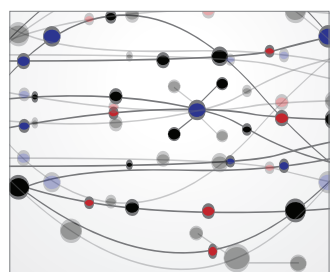

\section{The Scientific} World Journal
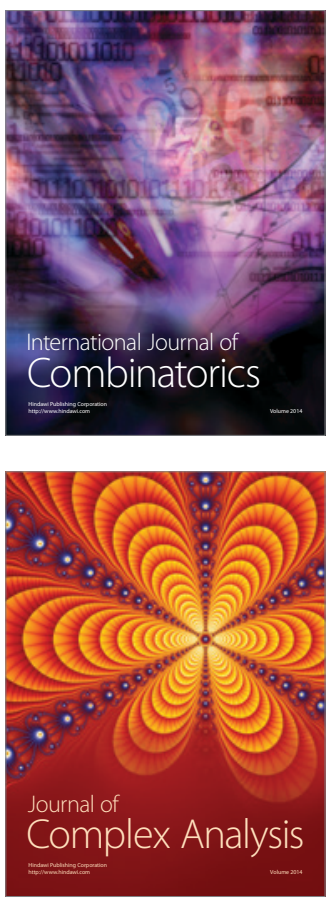

International Journal of

Mathematics and

Mathematical

Sciences
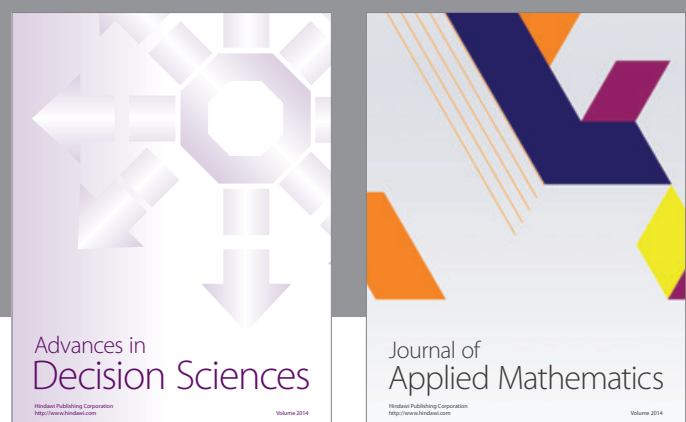

Journal of

Applied Mathematics
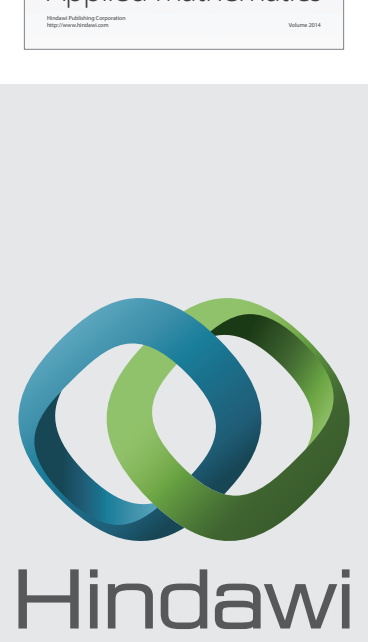

Submit your manuscripts at http://www.hindawi.com
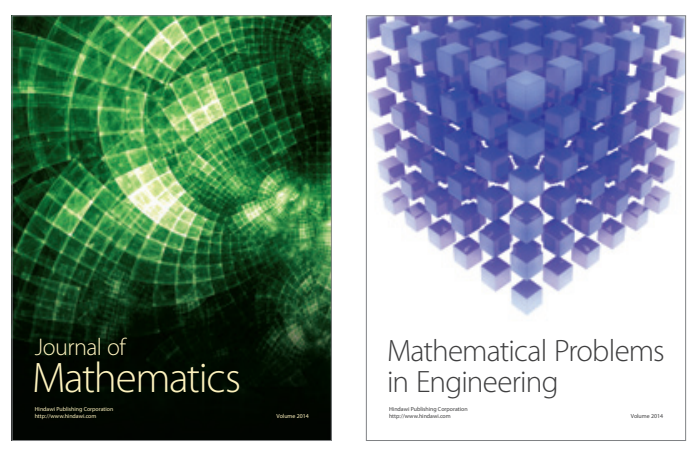

Mathematical Problems in Engineering
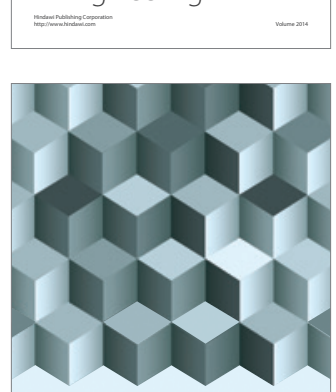

Journal of

Function Spaces
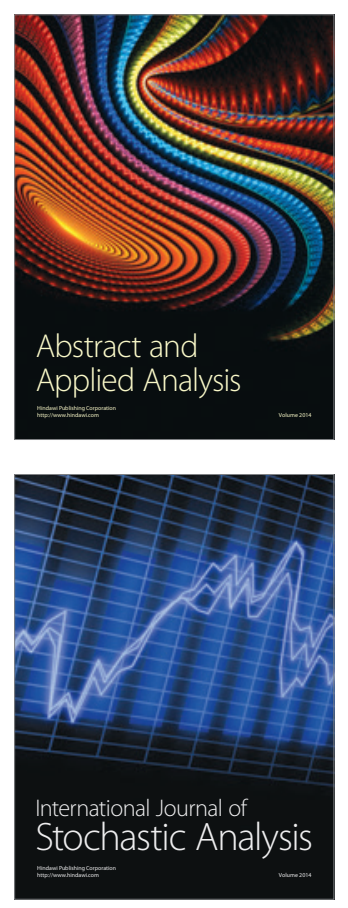

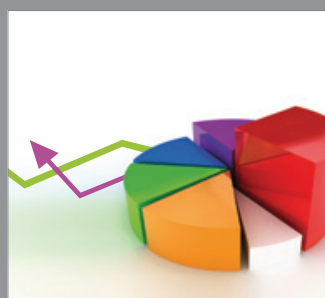

ournal of

Probability and Statistics

Promensencen
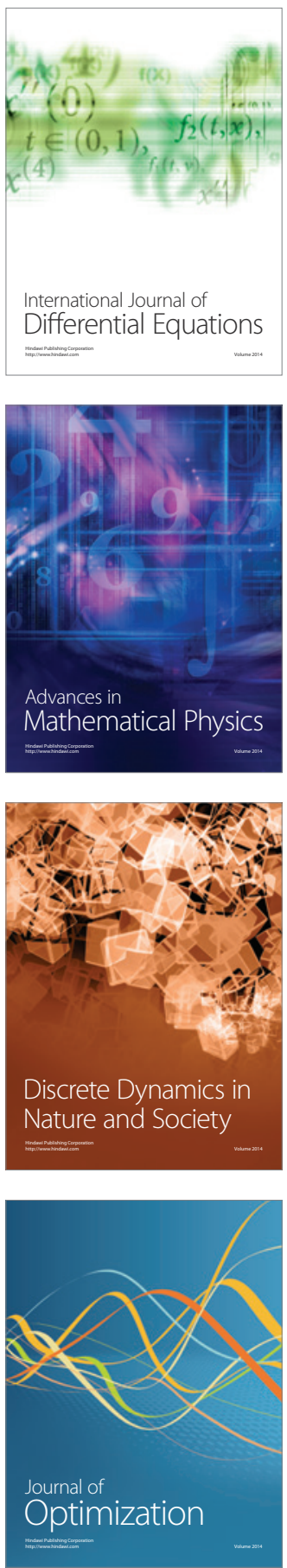